\title{
UNICORN Babies: Understanding Circulating and Cerebral Creatine Levels of the Preterm Infant. An Observational Study Protocol
}

OPEN ACCESS

Edited by:

Carina Mallard,

University of Gothenburg,

Sweden

Reviewed by:

Mhoyra Fraser,

The University of Auckland,

New Zealand

Michael Stark,

Women's and Children's Hospital,

Australia

*Correspondence:

Stacey J. Ellery

stacey.ellery@hudson.org.au

Specialty section:

This article was submitted to

Embryonic and Developmental

Physiology,

a section of the journal

Frontiers in Physiology

Received: 15 October 2018

Accepted: 07 February 2019

Published: 07 March 2019

Citation:

Berry MJ, Schlegel M, Kowalski GM,

Bruce CR, Callahan DL,

Davies-Tuck ML, Dickinson H, Goodson A, Slocombe A, Snow RJ, Walker DW and Ellery SJ (2019) UNICORN Babies: Understanding Circulating and Cerebral Creatine Levels of the Preterm Infant. An Observational Study Protocol. Front. Physiol. 10:142. doi: 10.3389/fphys.2019.00142
Mary J. Berry ${ }^{1,2}$, Melissa Schlegel ${ }^{1,2}$, Greg M. Kowalski ${ }^{3}$, Clinton R. Bruce ${ }^{3}$, Damien L. Callahan ${ }^{4}$, Miranda L. Davies-Tuck ${ }^{5}$, Hayley Dickinson ${ }^{5}$, Angus Goodson², Angie Slocombe ${ }^{2}$, Rod J. Snow ${ }^{3}$, David W. Walker ${ }^{6}$ and Stacey J. Ellery ${ }^{5 *}$

${ }^{1}$ Department of Paediatrics and Child Health, University of Otago, Wellington, New Zealand, ${ }^{2}$ Capital and Coast District Health Board, Wellington, New Zealand, ${ }^{3}$ School of Exercise Sciences, Institute for Physical Activity and Nutrition, Deakin University, Geelong, VIC, Australia, ${ }^{4}$ Centre for Cellular and Molecular Biology, School of Life and Environmental Science, Deakin University, Melbourne, VIC, Australia, ${ }^{5}$ The Ritchie Centre, Hudson Institute of Medical Research, and Department of Obstetrics and Gynaecology, Monash University, Clayton, VIC, Australia, ${ }^{6}$ School of Health and Biomedical Sciences, RMIT University, Melbourne, VIC, Australia

Creatine is an essential metabolite for brain function, with a fundamental role in cellular (ATP) energy homeostasis. It is hypothesized that preterm infants will become creatine deplete in the early postnatal period, due to premature delivery from a maternal source of creatine and a limited supply of creatine in newborn nutrition. This potential alteration to brain metabolism may contribute to, or compound, poor neurological outcomes in this high-risk population. Understanding Creatine for Neurological Health in Babies (UNICORN) is an observational study of circulating and cerebral creatine levels in preterm infants. We will recruit preterm infants at gestational ages $23^{+0}-26^{+6}, 27^{+0}-29^{+6}, 30^{+0}-32^{+6}$, $33^{+0}-36^{+6}$, and a term reference group at $39^{+0}-40^{+6}$ weeks of gestation, with 20 infants in each gestational age group. At birth, a maternal capillary blood sample, as well as a venous cord blood sample, will be collected. For preterm infants, serial infant plasma (heel prick), urine, and nutrition samples [total parenteral nutrition (TPN), breast milk, or formula] will be collected between birth and term "due date." Key fetomaternal information, including demographics, smoking status, and maternal diet, will also be collected. At term corrected postnatal age (CPA), each infant will undergo an MRI/ ${ }^{1} \mathrm{H}-\mathrm{MRS}$ scan to evaluate brain structure and measure cerebral creatine content. A general movements assessment (GMA) will also be conducted. At 3 months of CPA, infants will undergo a second GMA as well as further neurodevelopmental evaluation using the Developmental Assessment of Young Children - Second Edition (DAYC-2) assessment tool. The primary outcome measures for this study are cerebral creatine content at CPA and plasma and urine creatine and guanidinoacetate (creatine precursor) concentrations in the early postnatal period. We will also determine associations between (1) creatine levels at term CPA and neurodevelopmental outcomes (MRI, GMA, and DAY-C); (2) dietary creatine intake and circulating and cerebral creatine content; and (3) creatine levels and maternal 
characteristics. Novel approaches are needed to try and improve preterm-associated brain injury. Inclusion of creatine in preterm nutrition may better support ex utero brain development through improved cerebral cellular energy availability during a period of significant brain growth and development.

Ethics Ref: HDEC 18/CEN/7 New Zealand.

ACTRN: ACTRN12618000871246.

Keywords: premature, nutrition, brain metabolism, cellular energy, neurodevelopment

\section{INTRODUCTION}

The global incidence of preterm birth (delivery prior to 37 completed weeks of gestation) is reported to be $\sim 11 \%$ (Blencowe et al., 2013). Advances in perinatal care mean that increasing numbers of infants born even at extremes of gestational age (extreme preterm: $<28$ weeks of gestational age) survive beyond the neonatal period (Davidoff et al., 2006; Mohangoo et al., 2011; Lawn et al., 2013). Additionally, within resource-rich neonatal care settings, the age of human viability continues to fall with increasing survival rates at even 22 and 23 weeks of gestational age. However, this increase in survival has not been coupled with a reduction in long-term morbidity. Neurodevelopmental disorders, including those of executive function, psychiatric, and behavioral sequelae (Blencowe et al., 2013), are more prevalent in those born preterm than at term, with increasing risk, the lesser the gestational age at birth (Crump et al., 2010; Johnson et al., 2010).

The etiology of brain injury in preterm infants is complex and often unexplained. Historically, intraventricular hemorrhage (IVH) with resultant disruption of brain tissue and formation of macrocystic lesions was associated with global developmental delay. With the improvements in perinatal care, high grade IVH and/or periventricular leukomalacia (PVL) has become far less common, and standard cranial ultrasound or magnetic resonance imaging (MRI) examination usually do not demonstrate gross structural lesions (Penn et al., 2016). In Australia and New Zealand, the rate of high grade IVH in infants born prior to 32 weeks' gestation declined from $8 \%$ to just over $4 \%$ in the period between 2004 and 2013 (Chow et al., 2013). As such, longitudinal studies now indicate that a large percentage of children born preterm have neurodevelopmental problems in the absence of structural brain abnormalities detected with conventional imaging (Constable et al., 2008; Mullen et al., 2011; Aeby et al., 2013). These data suggest that conventional prognostication based on measures of cerebral structure is poorly discriminatory and will not always identify infants at risk of neurodevelopmental morbidity. We, and others, propose that our focus should be widened to explore the role of altered brain metabolism in the pathophysiology of preterm brain injury and neurodevelopmental disability (Koob et al., 2016).

Creatine is a metabolite essential for brain development and energy metabolism (Braissant et al., 2007). It is an amino acid derivative that fuels the creatine kinase circuit; a phosphagen system that mitigates temporal imbalances in energy (ATP) supply and demand (Wallimann et al., 1992). Via the creatine kinase circuit, creatine and its phosphorylated derivative (phosphocreatine, PCr) shuttle high-energy phosphate groups from the mitochondria into the cytosol, for the regeneration of ATP from ADP (Ellington, 2001). The creatine kinase isoforms required to run the circuit are found in the cerebellum, choroid plexus, hippocampal granular layer, and pyramidal cells of the brain (Hemmer et al., 1994) and are involved in maintaining ATP homeostasis in the central nervous system (CNS) from early in embryonic development through to adulthood (Braissant et al., 2001; Braissant et al., 2007).

Both creatine and phosphocreatine are spontaneously broken down into creatinine at a rate of $\sim 1.7 \%$ /day (Brosnan and Brosnan, 2007). Thus, there is a requirement to replenish creatine and PCr stores on a daily basis. Creatine can be obtained from the diet and is also endogenously synthesized de novo. Synthesis involves the production of guanidinoacetate (GAA) from arginine and glycine, in a reaction catalyzed by arginine:glycine aminotransferase (AGAT) that occurs predominately in the kidney and pancreas. GAA is then methylated by guanidinoacetate $\mathrm{N}$-methyltransferase (GAMT), mainly in the liver, to produce creatine. Once synthesized or absorbed, creatine is transported into cells via the sodiumdependent SLC6A8 creatine transporter (Wyss and KaddurahDaouk, 2000). Creatine is able to cross the blood-brain barrier (Ohtsuki et al., 2002), and the brain itself also has some inherent capacity to synthesize creatine, with both AGAT and GAMT expressed by neurons, astrocytes, and oligodendrocytes (Braissant et al., 2001; Tachikawa et al., 2004; Nakashima et al., 2005). Despite the importance of creatine for brain growth and development, the relative importance of cerebral creatine derived from systemic rather than local synthesis has not yet been established.

In utero fetal magnetic resonance spectroscopy ( $\left.{ }^{1} \mathrm{H}-\mathrm{MRS}\right)$ studies indicate cerebral creatine accretion occurs from 18 to 40 weeks of gestation (Evangelou et al., 2015). Postnatal studies have also clearly identified that an increase in cerebral creatine concentration occurs during the first 3 months of life (Blüml et al., 2012; Blüml et al., 2014). The importance of a maternal source of creatine for the developing fetus and the continued postnatal accretion of cerebral creatine is made evident by those infants diagnosed with inherited creatine deficiency syndromes. These infants typically raise no concerns antenatally; however, as postnatal creatine levels fall, they become progressively more symptomatic with altered neurological state 
(Almeida et al., 2006) and complex neurological symptoms including impaired psychomotor development, seizures, and cognitive impairment (Battini et al., 2006).

In adults, creatine is acquired via the diet and de novo synthesis, with each contributing 50\% (Brosnan and Brosnan, 2007). However, in infancy, due to low levels of creatine in human breast milk and commercial formulas, a term infant most likely synthesizes between 64 and 93\% of their daily creatine requirements (Edison et al., 2013). The age at which a fetus/newborn develops the capacity to synthesize creatine is currently unknown, but it requires sufficient renal, hepatic, and cerebral maturity to express the enzymes necessary for creatine synthesis. Evaluation of AGAT and GAMT expression in the rat embryo found that AGAT was expressed in isolated cells of the CNS from 0.6 gestation; however, widespread expression of AGAT and rudimentary GAMT expression were not apparent before 0.8 gestation (Braissant et al., 2005). Our own studies in the precocial spiny mouse found that the fetal kidney and liver do not express AGAT and GAMT before 0.9 gestation, an age and maturation equivalent to $\sim 35$ weeks' human gestation (Ireland et al., 2009). Taken together, these observations raise compelling questions about progressive creatine depletion in preterm infants, and whether this complication of nutritional compromise predisposes or compounds their risk of altered cerebral metabolism and thus long-term neurodevelopmental disability.

To date, a number of small observational studies have identified perturbations in creatine homeostasis in preterm infants (Blüml et al., 2014; Koob et al., 2016). Koob et al. (2016) reported that at term CPA creatine concentrations in the centrum semiovale of infants born preterm (born $29.1 \pm 2$ weeks) were reduced compared to those born at term (Koob et al., 2016). In addition, Lage et al. (2013) found that by hospital discharge, preterm infants had higher urinary GAA and reduced urinary creatine excretion, suggesting differences in systemic creatine synthesizing capacity. This was particularly apparent in their very preterm group (28-29 weeks) (Lage et al., 2013).

No studies have characterized longitudinal changes in creatine metabolism among preterm infants or the association between cerebral creatine content and neurodevelopmental outcomes. Further to this, no studies have monitored systemic creatine levels (both circulating and excreted) in a single preterm population nor have they assessed nutritional creatine availability for preterm infants fed with total parenteral nutrition (TPN), preterm infant formulas, or breast milk.

We hypothesize that following preterm birth, peripheral and cerebral creatine levels will fall, so that at term CPA, preterm infants will have relative systemic and cerebral creatine deficiency. Furthermore, this reduction in creatine bioavailability will jeopardize normal brain metabolism and development, ultimately predisposing the infant to neurodevelopmental dysfunction.

The overall aim of this study is to assess whether preterm infants become creatine deplete in the early postnatal period, by measuring the preterm infants' circulating and excreted levels of creatine and GAA and comparing these measures to cerebral creatine content, as determined by ${ }^{1} \mathrm{H}-\mathrm{MRS}$, at term corrected age. This study will correlate preterm creatine levels with neurological outcomes using MRI, general movements assessment (GMA), and the Developmental Assessment of Young Children - Second Edition (DAYC-2) Test (Novak et al., 2017). This study will also establish the levels of creatine provided through dietary intake during the days and weeks after preterm birth. Results of this study may support the use of creatine supplementation as standard nutritional care of the preterm infant, in order to improve neurodevelopmental function in this vulnerable population.

\section{Specific Aims}

1. Compare cerebral, plasma, and urine creatine and GAA content between preterm and term infants at CPA.

2. Describe preterm infant plasma and urine creatine and GAA concentrations and creatine:GAA ratio in the early postnatal period.

3. Describe associations between cerebral creatine content, plasma, and urine creatine concentrations with maternal and infant characteristics at CPA.

4. Compare creatine, GAA, arginine, glycine, and methionine concentrations in standard preterm nutrition, including TPN, preterm infant formulas, and breast milk.

5. Determine whether dietary source of creatine (TPN, formula, or breast milk) is related to plasma or urine creatine concentrations in the early postnatal period.

6. Describe associations between cerebral creatine content and blood and urine creatine concentrations with neurological outcomes (GMA, MRI, and DAYC-2) at CPA and at 3 months of corrected postnatal age.

\section{METHOD/DESIGN}

\section{Study Design}

Prospective, single-center, observational study.

\section{Study Setting}

Neonatal Intensive Care Unit and Postnatal Wards, Capital Coast District Hospital Board (CCDHB), Wellington, New Zealand.

\section{Primary Outcome Measures}

1. Cerebral creatine content at term CPA

2. Plasma (bi-weekly) and urine (weekly) creatine and GAA levels from birth until hospital discharge and at CPA

3. Creatine, GAA, and amino acid levels in infant nutrition (TPN, formula, or breast milk) supplied from birth until discharge and at CPA

\section{Secondary Outcome Measures}

1. Global injury score from MRI at term CPA

2. General movement assessment score at term CPA and 3 months of CPA

3. DAYC-2 assessment score at 3 months of CPA 


\section{Study Population}

\section{Inclusion Criteria}

1. Preterm infants receiving care within the Neonatal Intensive Care Unit at CCDHB or healthy term infants at 39-40 weeks' gestation from low-risk pregnancies delivered at CCDHB.

\section{Exclusion Criteria}

1. Babies with major congenital, genetic, or chromosomal abnormalities known to affect neurodevelopmental outcomes

2. Hypoxic ischemic encephalopathy

3. Inborn (proven or suspected) errors of metabolism

4. Fetal growth restriction (birth weight $<10$ th percentile on customized percentiles)

\section{Recruitment}

We will recruit infants at gestational ages $23^{+0}-26^{+6}, 27^{+0}-29^{+6}$, $30^{+0}-32^{+6}, 33^{+0}-36^{+6}$, and $39^{+0}-40^{+6}$ weeks. We will aim to capture 20 infants at each time point (with equal numbers of males and females). Eligible infants will be identified based on gestational age at delivery and consideration of inclusion and exclusion criteria. Pregnant women will be approached at presentation to Delivery Suite to discuss study participation. If consent is obtained, the mother and infant will be recruited to the study and the consulting obstetric, midwifery, neonatal, and nursing team advised of their involvement. Where pre-delivery consent is not obtained due to rapidity of labor and/or other clinical factors, cord blood will be collected preemptively, and retrospective consent and maternal blood sample sought within $24 \mathrm{~h}$ of delivery. If the parents do not wish to participate, the cord blood sample will be disposed of using routine clinical biological waste disposal systems and no further study samples collected.

\section{Sample and Data Collection}

\section{Blood, Urine, and Nutrition Sampling}

A schematic overview of the sampling regime for each participant is outlined in Figure 1. At the time of birth, we will use a finger prick to collect $\sim 120 \mu$ l of maternal whole blood using a capillary tube. A 2-ml venous cord blood sample will also be collected from which a $\sim 120 \mu$ l sample will be obtained using a capillary tube. Then, while infants are receiving care in the NICU, or on postnatal wards, and at follow-up appointments, the following samples will be collected. Sample frequency will be dictated by gestational age at birth.

Blood sampling: A blood sample will be collected biweekly. We will perform a heel prick to collect $\sim 120 \mu$ l of whole blood using a capillary tube. All blood samples (cord, maternal, or infant) will be transferred to a Hemaspot ${ }^{\mathrm{TM}} \mathrm{SE}$ blood separation device to facilitate collection of the cell-free blood fraction (referred to throughout as plasma for clarity) from a small volume of whole blood. Hemaspots are allowed to dry at room temperature before being stored at $-20^{\circ} \mathrm{C}$ for future analysis.

Urine sampling: Around the same time as the blood collection, and on each alternate week, we will place some gauze into the infant's nappy to facilitate a spot urine collection. Once obtained, this sample will be transferred to an Eppendorf tube and stored at $-80^{\circ} \mathrm{C}$ for future analysis.

Nutrition sampling: A $200 \mu \mathrm{l}$ sample of the nutrition (TNP, formula, and breast milk) being fed to each infant at the time of the blood and urine sampling will be collected. If in powdered form (formula), this will be reconstituted following standard practices. All samples will be transferred into an Eppendorf tube and stored at $-80^{\circ} \mathrm{C}$ for future analysis.

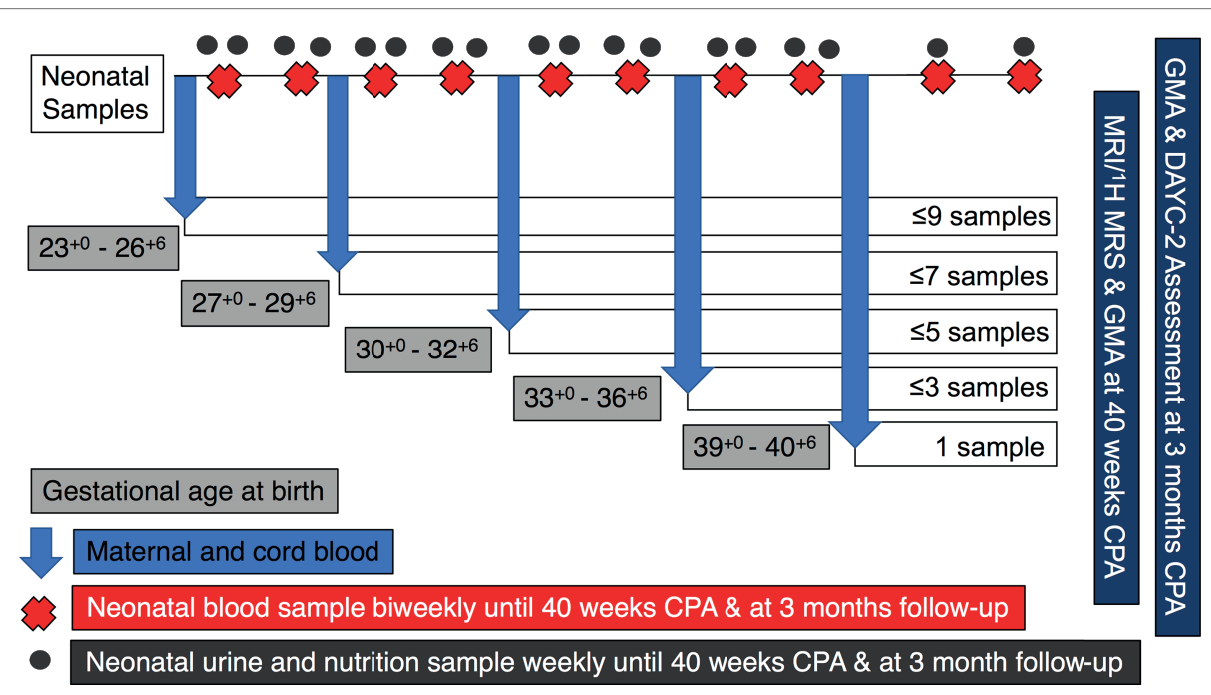

FIGURE 1 | Schematic representation of UNICORN sampling regime. Samples of neonatal blood, urine, and nutrition will be collected weekly or bi-weekly for the duration of the infants stay in hospital. Magnetic resonance imaging (MRI) and magnetic resonance spectroscopy $\left({ }^{1} \mathrm{H}-\mathrm{MRS}\right)$ will be performed at term corrected postnatal age (CPA). Infants will also undergo a general movements assessment (GMA) at CPA and at 3 months CPA. At the follow-up appointment at 3 months CPA, further neurological assessment will be completed using the Developmental Assessment of Young Children - Second Edition (DAYC-2) assessment tool. 


\section{Monitoring and Data Collection}

The following covariates/potential confounders will be collected as part of this study:

Maternal characteristics and birth outcomes data:

- Maternal age

- Comorbidities/factors (preeclampsia, gestational diabetes, chorioamnionitis, IVF)

- Country of birth

- Dietary preferences (omnivorous, vegetarian, vegan)

- Smoking status

- Parity

- Gravity

- Mode of delivery

- Placental weight

Infant characteristics:

- Gestational age

- Sex

- Birth weight

- Ethnicity

- Weight and corrected postnatal age at the time of each sample collection

- Presence of postnatal infection (blood culture positive and/ or treatment with systemic antibiotics for $>24 \mathrm{~h}$ ) or necrotizing enterocolitis (Modified Bells Classification Grade II-III determined by the treating Pediatric Surgeon at time of diagnosis).

- Feeding schedule prior to each sample collection $(<24 \mathrm{~h})$

- Venous hemoglobin and hematocrit count prior to blood sample collection

\section{MRI/ ${ }^{1} \mathrm{H}-\mathrm{MRS}$ Data Acquisition}

At 40 weeks of corrected postnatal age (CPA), each participant will undergo a MRI/ ${ }^{1} \mathrm{H}-\mathrm{MRS}$ scan. MR examinations will be performed on a Siemens 3T Skyra using a 16-channel dedicated pediatric head coil. Infants will be examined without sedation using a "feed and wrap" technique according to standard institutional practice. In brief, infants are brought to the MRI unit, fed, and swaddled comfortably while being monitored with continuous pulse oximetry before being placed in the MRI scanner. Standard, size appropriate, hearing protection will be used for all infants. Sequences will be performed to demonstrate brain anatomy and will include T1 3D $1 \mathrm{~mm}$ volumetric, T2 $1 \mathrm{~mm} \mathrm{3D}$ volumetric scans, T2 TSE FLAIR, and T1SE MTC sequences. These sequences will also contribute to the analysis of any detected brain pathology. T2 SWI axial images will be performed to show calcification and blood products. Sequences for the detection of vascular injury and infarction will include diffusion sequences (b value $=2000$ ), resting BOLD, and arterial spin labeling. ${ }^{1} \mathrm{H}$-MRS will provide chemical spectra of the brain to further analyze brain pathology and measurement of cerebral creatine content. For MR spectroscopy, the voxel will be placed over the white matter underlying the central sulcus within the centrum semiovale and spectra acquired with a long echo time (TE $135 \mathrm{~ms}$ ) (Koob et al., 2016).

\section{Neurodevelopmental Observations}

At term $\mathrm{CPA}$, prior to the $\mathrm{MRI} /{ }^{1} \mathrm{H}-\mathrm{MRS}$ scan, an experienced neurodevelopmental therapist will record a 5-minute video of the infant to conduct a general movements assessment (GMA); a validated tool to noninvasively identify neurological issues, which may lead to cerebral palsy and other developmental disabilities (Spittle et al., 2018). Infants are placed in a supine position, with minimal clothing to allow freedom of movement. The video will be taken when the infant is fully alert, but not directly interacting with parents, healthcare providers, or receiving stimulus from any external sources.

At 3 months of CPA, we will request participants meet again with a developmental therapist. At this appointment, information about general health (including weight gain trajectories, feeding habits, and number of visits to a GP) will be recorded. A questionnaire about developmental milestones at 3 months of age (developed by the American Academy of Pediatrics) will be completed, as well as a Developmental Assessment of Young Children - Second Edition (DAYC-2) Test, a validated tool to identify children with possible developmental delay in the following domains: cognition, communication, social-emotional development, physical development, and adaptive behavior (Saleh and Smadi, 2017). A 5-minute video of the infant will also be recorded to allow for a second GMA to be completed.

\section{Sample and Data Analysis \\ Creatine, GAA, and Amino Acid Analysis}

Creatine, GAA, arginine, glycine, and methionine will be measured in biological and nutrition samples via LC-MS/MS, according to the methods of (Tran et al., 2014), with slight modification (Tran et al., 2014). Briefly, a 6-mm hole punch of the "cell-free" plasma fraction of the Hemaspot ${ }^{\mathrm{TM}}, 7 \mu \mathrm{l}$ of urine, nutrition, or unlabeled standard, will be transferred to $1.5-\mathrm{ml}$ safety lock Eppendorf tubes. $200 \mu \mathrm{l}$ of methanol containing $10 \mu \mathrm{M} 2,2-\mathrm{d}_{3}$ Creatine (Sigma), $2.5 \mu \mathrm{M} 2,2-\mathrm{d}_{2}$ GAA (Sigma), and $2.5 \mu \mathrm{M}$ of Labeled Amino Acid Standard Set A (Cambridge Isotope Laboratories Inc.) internal standards is added to each tube. Samples are sonicated for $5 \mathrm{~min}$ then vortexed for $20 \mathrm{~min}$, before being centrifuged at 20,000x $\mathrm{g}$ for $5 \mathrm{~min}$ to pellet any precipitate or filter paper at the bottom of the tube. Supernatant $(\sim 170 \mu \mathrm{l})$ is transferred into $250-\mu \mathrm{l}$ glass inserts placed in 2-ml glass vials (Agilent), followed by evaporation to complete dryness in a speed vacuum (Labconco, Kansas, MO, USA). After drying, the GAA and creatine carboxyl groups are derivatized (butylated) by the addition of $100 \mu \mathrm{l}$ of $3 \mathrm{M}$ butanol-HCL followed by incubation at $60^{\circ} \mathrm{C}$ for $30 \mathrm{~min}$. After cooling to room temperature, excess butanol-HCL is evaporated under speed vacuum, after which the dry residue is resuspended in $200 \mu \mathrm{l}$ of methanol: water $(1: 1 \mathrm{v} / \mathrm{v})$ solution prior to LC-MS/MS analysis. The external standards consist of an eight sample serial dilution series of known concentration range $(0-25 \mu \mathrm{M}$ unlabeled GAA and $0-100 \mu \mathrm{M}$ unlabeled creatine).

The LC-MS/MS system is composed of a vacuum degasser, binary pumps, column oven, and a temperature-controlled autosampler (Shimadzu, Nexera ${ }^{\circledR}$ UPLC). This will be interfaced with a triple quadrupole mass spectrometer (Shimadzu, LCMS8030) with electrospray ionization. The LC column (Agilent 
$-2.1 \times 100 \mathrm{~mm}, 1.8 \mu \mathrm{m}$ C18 Zorbax Eclipse plus) will be maintained at $30^{\circ} \mathrm{C}$ and elution carried out with a binary gradient

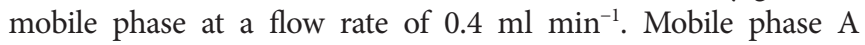
will contain water with $0.1 \%$ formic acid, and mobile phase $\mathrm{B}$ will contain acetonitrile with $0.1 \%$ formic acid. The initial mobile phase composition will be $5 \% \mathrm{~B}$, which is ramped linearly to $50 \%$ B over 6 min followed by a 2 -min column wash at $100 \%$ $\mathrm{B}$, with the column finally undergoing re-equilibration at $5 \% \mathrm{~B}$ for $3 \mathrm{~min}$, thus yielding a total runtime of $11 \mathrm{~min}$. The first $1 \mathrm{~min}$ is diverted to waste. The following ESI source conditions will be used: nebulizer gas flow rate $3 \mathrm{~L} \mathrm{~min}^{-1}$, desolvation line temperature at $250^{\circ} \mathrm{C}$, heater block temperature $400^{\circ} \mathrm{C}$, and drying gas flow rate of $15 \mathrm{~L} \mathrm{~min}^{-1}$. The MS will be operated in positive ionization with multiple reaction monitoring (MRM). The collision energy, Q1 and Q3 pre-bias voltages for the MRM transitions have been optimized using authentic creatine and GAA standards (SIGMA) via the automatic optimisation software (Labsolutions, Shimadzu), with optimal collision energy for creatine and GAA for the instrument being $-15 \mathrm{eV}$. The precursor and product ion transitions were creatine 188.2-90 m/z, $\mathrm{d}_{3}$-Creatine $191.2-93 \mathrm{~m} / z$, GAA $174.2-101.1 \mathrm{~m} / z$, and $\mathrm{d}_{2}$-GAA $176.1-103.1 \mathrm{~m} / \mathrm{z}$. The sample and standard injection volume will be $5 \mu \mathrm{l}$. Peak areas will be determined using Lab solutions post-run browser software (Shimadzu). The creatine, GAA and amino acid concentrations of blood spots, urine, or nutrition samples will be calculated via linear regression of the serially diluted external (unlabeled) standard series using the isotope dilution technique.

\section{MRI/ ${ }^{1} \mathrm{H}-\mathrm{MRS}$}

MRI images will be scored according to the Kidokoro assessment tool (Kidokoro et al., 2013). Briefly, cerebral white matter, cortical gray matter, deep gray matter, and cerebellar abnormalities will be independently graded, and then the scores combined to calculate a single global brain injury score. The assessor will also note the presence of hemorrhage or cystic lesions throughout the brain. Hemorrhagic lesions will be graded using the Papile classification system (Papile et al., 1978) and cystic lesions characterized as periventricular leukomalacia, periventricular hemorrhagic infarction, or other (Barkovich, 2000).

Metabolic ratios from ${ }^{1} \mathrm{H}$-MRS spectra obtained with a TE of $135 \mathrm{~ms}$ will be calculated as previously described, with water used as an internal reference to normalize metabolite signal intensities (Koob et al., 2016).

\section{Neurodevelopmental Observations}

Motor function will be assessed by a developmental therapist trained in GMA and blinded to the clinical history of the infant. At term CPA, infants will be assessed on withering movements, which should include complex movements of the arms, legs, neck, and trunk. Poor withering movements include rigid or chaotic movements that lack fluency and coordination (Spittle et al., 2013) and are predictive of adverse neurodevelopmental outcomes. At 3 months of CPA, infants will be assessed on fidgety movements, which appear as small continual movements of limbs in all directions. Poor quality fidgety movements include larger amplitude and jerking movements (Spittle et al., 2013) and are again predictive of later outcomes. Infants will be scored independently at each postnatal age.

At 3-month CPA, infants' development will also be reviewed using the DAYC-2 assessment tool (Saleh and Smadi, 2017). An overall score from the five domains tested (cognition, communication, social-emotional development, physical development, and adaptive behavior) will be generated for each infant. Collectively, MRI, GMA, and DAYC-2 scores will be used to assess the potential risk of poor neurological outcomes, based on early diagnosis strategies for cerebral palsy (Novak et al., 2017).

\section{Sample Size}

This study will be the first observational study of creatine homeostasis in the preterm infant that encompasses plasma, urine, nutrition, and cerebral creatine measures across time. As the outcomes are mainly descriptive, no formal power calculation has been performed. However, ${ }^{1} \mathrm{H}-\mathrm{MRS}$ results reported by Koob et al. (2016) showed comparison of 13 preterm vs term infants allowed for measurement of a statistically significant $(-17 \%)$ reduction in cerebral creatine content in preterm infants at $\mathrm{CPA}\left(\mathrm{tCr} / \mathrm{H}_{2} \mathrm{O} \times 10^{-3}\right.$; term $0.12 \pm 0.1$ vs preterm $0.10 \pm 0.1$ ). This same study had a $20-25 \%$ failure rate in obtaining accurate $\mathrm{MRI} /{ }^{1} \mathrm{H}$-MRS measurements at $\mathrm{CPA}$ (Koob et al., 2016). Taking these data into consideration and a potential loss to follow-up at 3-month of $20 \%$, we aim to capture 20 infants in each gestational group.

\section{Statistical Analysis}

Characteristics of the population will be tabulated, along with appropriate descriptive statistics of the study sample. Continuous data will be assessed for normality and expressed as means and standard deviations or median and interquartile range as appropriate. Categorical data will be expressed as counts and proportions. Average creatine content from nutrition per week for each baby in the early postnatal period will be calculated.

Differences in creatine and GAA measures $\left({ }^{1} \mathrm{H}-\mathrm{MRS}\right.$, plasma or urine) between infants born preterm and those born at term will be assessed at CPA using a t-test or Mann-Whitney $\mathrm{U}$ test as appropriate. The correlation between gestational age at birth and creatine and GAA measures at CPA will also be determined using a Spearman or Pearson correlation. Preterm infant plasma and urine creatine and GAA concentrations and creatine:GAA ratio in the early postnatal period will be described and plotted. Associations between cerebral creatine content, plasma and urine creatine concentrations with maternal, and infant characteristics at CPA will be determined using Spearman or Pearson correlations for continuous data (maternal age, gestational age, birth weight, parity, gravity, placental weight) or a t-test or Mann-Whitney $U$ test for categorical data (infant sex, mode of delivery, maternal comorbidities, country of birth, ethnicity, and dietary preferences), as appropriate. Differences in creatine, GAA, and amino acid content of nutrition sources (TNP, formula or breast milk) will be determined using a one-way ANOVA or Kruskal-Wallis $\mathrm{H}$ test as appropriate. The association between creatine, GAA, and amino acid content of nutrition sources (TNP, formula 
or breast milk) and infant plasma and urine creatine at CPA will be assessed using a Spearman or Pearson correlation as appropriate. Finally, the association between cerebral creatine content, plasma creatine levels, and the ratio of plasma:cerebral creatine at CPA with GMA, MRI global injury, and DAYC-2 scores will be determined using linear regression at both CPA and at 3 months' corrected postnatal age. Potential confounders such as gestational age and maternal and infant characteristics will be assessed, along with collinearity, prior to performing multivariable linear regression.

\section{DISCUSSION}

Both creatine and the creatine kinase circuit are critical for brain metabolism (Braissant et al., 2011). It is hypothesized that preterm infants will become creatine deplete in the early postnatal period, due to premature delivery from a maternal source of creatine in utero, limited capacity for endogenous synthesis postnatally, and a limited supply of creatine in newborn nutrition. This creatine depletion may alter cerebral creatine metabolism, contributing to or compounding the risk of neurological disability in this population.

We anticipate that preterm babies will have altered creatine homeostasis and reduced creatine accretion in the early postnatal period compared to an infant of the same postconceptional age. Whether or not relative creatine deficiency is associated with neurological deficit will be a secondary outcome of this study. As the results are mainly descriptive and a formal power calculation could not be completed, there

\section{REFERENCES}

Aeby, A., De Tiège, X., Creuzil, M., David, P., Balériaux, D., Van Overmeire, B., et al. (2013). Language development at 2 years is correlated to brain microstructure in the left superior temporal gyrus at term equivalent age: a diffusion tensor imaging study. Neuroimage 78, 145-151. doi: 10.1016/j. neuroimage.2013.03.076

Almeida, L. G. S., Rosenberg, E. H., Verhoeven, N. M., Jakobs, C., and Salomons, G. S. (2006). Are cerebral creatine deficiency syndromes on the radar screen? Future Neurol. 1, 637-649. doi: 10.2217/14796708.1.5.637

Barkovich, A. J. (2000). Brain and spine injury in infancy and childhood. J. Pediatr. Neurol. 30, 157-250.

Battini, R., Alessandri, M., Leuzzi, V., Moro, F., Tosetti, M., Bianchi, M., et al. (2006). Arginine:glycine amidinotransferase (AGAT) deficiency in a newborn: early treatment can prevent phenotypic expression of the disease. J. Pediatr. 148, 828-830. doi: 10.1016/j.jpeds.2006.01.043

Blencowe, H., Cousens, S., Chou, D., Oestergaard, M., Say, L., Moller, A. -B., et al. (2013). Born too soon: the global epidemiology of 15 million preterm births. Reprod. Health 10:S2. doi: 10.1186/1742-4755-10-S1-S2

Blüml, S., Wisnowski, J. L., Nelson, M. D. Jr., Paquette, L., Gilles, F. H., Kinney, H. C., et al. (2012). Metabolic maturation of the human brain from birth through adolescence: insights from in vivo magnetic resonance spectroscopy. Cereb. Cortex 23, 2944-2955. doi: 10.1093/cercor/bhs283

Blüml, S., Wisnowski, J. L. Nelson, M. D. Jr., Paquette, L., and Panigrahy, A. (2014). Metabolic maturation of white matter is altered in preterm infants. PLoS One 9:E85829. doi: 10.1371/journal.pone.0085829

Braissant, O., Bachmann, C., and Henry, H. (2007). "Expression and function of AGAT, GAMT and CT1 in the mammalian brain" in Creatine and creatine kinase in health and disease. Subcellular Biochemistry. eds. G. S. Salomons, and M. Wyss vol 46, (Dordrecht: Springer). doi: 10.1007/978-1-4020-6486-9_4 is the possibility that sample size may become a limitation for some of the proposed analyses in this observational study. However, the data obtained will enable calculation of effect size for future studies. If our overall hypothesis is supported, this observational study will inform a future randomized control trial of infant dietary creatine supplementation following premature birth, with the aim of improving brain metabolism during a key stage of development and thereby reducing the burden of neurodevelopmental disability in this highrisk population.

\section{AUTHOR CONTRIBUTIONS}

$\mathrm{MB}, \mathrm{SE}, \mathrm{HD}$, and DW conceptualized the study. MS is a research nurse coordinating recruitment, sample collection, and data management. AG was involved in ethics development and study design. GK, CB, DC, and RS developed the LC-MS/ MS protocol for creatine and metabolite assessment. AS developed the MR data acquisition protocol. MD-T designed the statistical approaches to the study. $\mathrm{MB}$ and SE wrote the manuscript. All authors contributed to the drafting of the manuscript.

\section{FUNDING}

The authors would like to acknowledge funding support from the Cerebral Palsy Alliance (PG2715) and Rebecca L Cooper Medical Research Foundation (10624) for the completion of this study.

Braissant, O., Henry, H., Béard, E., and Uldry, J. (2011). Creatine deficiency syndromes and the importance of creatine synthesis in the brain. Amino Acids 40, 1315-1324. doi: 10.1007/s00726-011-0852-Z

Braissant, O., Henry, H., Loup, M., Eilers, B., and Bachmann, C. (2001). Endogenous synthesis and transport of creatine in the rat brain: an in situ hybridization study. Mol. Brain Res. 86, 193-201. doi: 10.1016/S0169-328X(00)00269-2

Braissant, O., Henry, H., Villard, A. -M., Speer, O., Wallimann, T., and Bachmann, C. (2005). Creatine synthesis and transport during rat embryogenesis: spatiotemporal expression of AGAT, GAMT and CT1. BMC Dev. Biol. 5:9. doi: 10.1186/1471-213X-5-9

Brosnan, J., and Brosnan, M. (2007). Creatine: endogenous metabolite, dietary, and therapeutic supplement. Annu. Rev. Nutr. 27, 241-261. doi: 10.1146/ annurev.nutr.27.061406.093621

Chow, S., Le Marsney, R., Hossain, S., Haslam, R., and Lui, K. (2013). Report Of The Australian And New Zealand Neonatal Network 2012. Sydney: Anznn.

Constable, R. T., Ment, L. R., Vohr, B. R., Kesler, S. R., Fulbright, R. K., Lacadie, C., et al. (2008). Prematurely born children demonstrate white matter microstructural differences at 12 years of age, relative to term control subjects: an investigation of group and gender effects. Pediatrics 121, 306-316. doi: 10.1542/peds.2007-0414

Crump, C., Winkleby, M. A., Sundquist, K., and Sundquist, J. (2010). Preterm birth and psychiatric medication prescription in young adulthood: a Swedish national cohort study. Int. J. Epidemiol. 39, 1522-1530. doi: 10.1093/ije/ dyq103

Davidoff, M. J., Dias, T., Damus, K., Russell, R., Bettegowda, V. R., Dolan, S., et al. (2006). Changes in the gestational age distribution among us singleton births: impact on rates of late preterm birth, 1992 to 2002. Semin. Perinatol. 30, 8-15. doi: 10.1053/j.semperi.2006.01.009

Edison, E. E., Brosnan, M. E., Aziz, K., and Brosnan, J. T. (2013). Creatine and guanidinoacetate content of human milk and infant formulas: implications 
for creatine deficiency syndromes and amino acid metabolism. Br. J. Nutr. 110, 1075-1078. doi: 10.1017/S000711451300010X

Ellington, W. R. (2001). Evolution and physiological roles of phosphagen systems. Annu. Rev. Physiol. 63, 289-325. doi: 10.1146/annurev.physiol.63.1.289

Evangelou, I. E., Du Plessis, A. J., Vezina, G., Noeske, R., and Limperopoulos, C. (2015). Elucidating metabolic maturation in the healthy fetal brain using $1 \mathrm{~h}$ $\mathrm{mr}$ spectroscopy. Am. J. Neuroradiol. doi: 10.3174/ajnr.A4512

Hemmer, W., Zanolla, E., Furter-Graves, E. M., Eppenberger, H. M., and Wallimann, T. (1994). Creatine kinase isoenzymes in chicken cerebellum: specific localization of brain-type creatine kinase in Bergmann glial cells and muscle-type creatine kinase in Purkinje neurons. Eur. J. Neurosci. 6, 538-549. doi: 10.1111/j.1460-9568.1994.tb00298.x

Ireland, Z., Russell, A., Wallimann, T., Walker, D. and Snow, R. (2009). Developmental changes in the expression of creatine synthesizing enzymes and creatine transporter in a precocial rodent, the spiny mouse. BMC Dev. Biol. 9, 39-50. doi: 10.1186/1471-213X-9-39

Johnson, S., Hollis, C., Kochhar, P., Hennessy, E., Wolke, D., and Marlow, N. (2010). Psychiatric disorders in extremely preterm children: longitudinal finding at age 11 years in the epicure study. J. Am. Acad. Child Adolesc. Psychiatry 49, 453-463.e1. doi: 10.1016/j.jaac.2010.02.002

Kidokoro, H., Neil, J., and Inder, T. (2013). New MR imaging assessment tool to define brain abnormalities in very preterm infants at term. Am. J. Neuroradiol. doi: 10.1681/ASN.2012080783

Koob, M., Viola, A., Le Fur, Y., Viout, P., Ratiney, H., Confort-Gouny, S., et al. (2016). Creatine, glutamine plus glutamate, and macromolecules are decreased in the central white matter of premature neonates around term. PLoS One 11:E0160990. doi: 10.1371/journal.pone.0160990

Lage, S., Andrade, F., Prieto, J. A., Asla, I., Rodríguez, A., Ruiz, N., et al. (2013). Arginine-guanidinoacetate-creatine pathway in preterm newborns: creatine biosynthesis in newborns. J. Pediatr. Endocrinol. Metab. 26, 53-60. doi: 10.1515/jpem-2012-0293

Lawn, J. E., Davidge, R., Paul, V. K., Von Xylander, S., De Graft Johnson, J., Costello, A., et al. (2013). Born too soon: care for the preterm baby. Reprod. Health 10:S5. doi: 10.1186/1742-4755-10-S1-S5

Mohangoo, A. D., Buitendijk, S. E., Szamotulska, K., Chalmers, J., Irgens, L. M., Bolumar, F., et al. (2011). Gestational age patterns of fetal and neonatal mortality in Europe: results from the Euro-Peristat project. PLoS One 6:E24727. doi: 10.1371/journal.pone.0024727

Mullen, K. M., Vohr, B. R., Katz, K. H., Schneider, K. C., Lacadie, C., Hampson, M., et al. (2011). Preterm birth results in alterations in neural connectivity at age 16 years. NeuroImage 54, 2563-2570. doi: 10.1016/j.neuroimage.2010.11.019

Nakashima, T., Tomi, M., Tachikawa, M., Watanabe, M., Terasaki, T., and Hosoya, K. I. (2005). Evidence for creatine biosynthesis in Müller glia. Glia 52, 47-52. doi: 10.1002/glia.20222

Novak, I., Morgan, C., Adde, L., Blackman, J., Boyd, R. N., Brunstrom-Hernandez, J., et al. (2017). Early, accurate diagnosis and early intervention in cerebral palsy: advances in diagnosis and treatment. JAMA Pediatr. 171, 897-907. doi: $10.1001 /$ jamapediatrics.2017.1689

Ohtsuki, S., Tachikawa, M., Takanaga, H., Shimizu, H., Watanabe, M., Hosoya, K. -I., et al. (2002). The blood-brain barrier creatine transporter is a major pathway for supplying creatine to the brain. J. Cereb. Blood Flow Metab. 22, 1327-1335. doi: 10.1097/01.WCB.0000033966.83623.7D

Papile, L. -A., Burstein, J., Burstein, R., and Koffler, H. (1978). Incidence and evolution of subependymal and intraventricular hemorrhage: a study of infants with birth weights less than 1,500 Gm. J. Pediatr. 92, 529-534. doi: 10.1016/S0022-3476(78)80282-0

Penn, A. A., Gressens, P., Fleiss, B., Back, S. A., and Gallo, V. (2016). Controversies in preterm brain injury. Neurobiol. Dis. 92, 90-101. doi: 10.1016/j. nbd.2015.10.012

Saleh, R. M. A., and Smadi, J. M. (2017). The efficacy of arabic version of the developmental assessment of young children second edition (Dayc-2) scale in detecting developmental delay among Jordanian children aged birth to 71 months. Int. Educ. Stud. 10, 113-132. doi: 10.5539/ies.v10n4p113

Spittle, A. J., Morgan, C., Olsen, J. E., Novak, I., and Cheong, J. L. (2018). Early diagnosis and treatment of cerebral palsy in children with a history of preterm birth. Clin Perinatol. doi: 10.1111/jpc.14149

Spittle, A. J., Spencer-Smith, M. M., Cheong, J. L., Eeles, A. L., Lee, K. J., Anderson, P. J., et al. (2013). General movements in very preterm children and neurodevelopment at 2 and 4 years. Pediatr. Peds. 45. doi: 10.1016/j. clp.2018.05.011

Tachikawa, M., Fukaya, M., Terasaki, T., Ohtsuki, S., and Watanabe, M. (2004). Distinct cellular expressions of creatine synthetic enzyme gamt and creatine kinases UCK-MI And CK-B suggest a novel neuron-glial relationship for brain energy homeostasis. Eur. J. Neurosci. 20, 144-160. doi: 10.1111/j.1460-9568.2004.03478.x

Tran, C., Yazdanpanah, M., Kyriakopoulou, L., Levandovskiy, V., Zahid, H., Naufer, A., et al. (2014). Stable isotope dilution microquantification of creatine metabolites in plasma, whole blood and dried blood spots for pharmacological studies in mouse models of creatine deficiency. Clin. Chim. Acta 436, 160-168. doi: 10.1016/j.cca.2014.05.007

Wallimann, T., Wyss, M., Brdiczka, D., Nicolay, K., and Eppenberger, H. (1992). Intracellular compartmentation, structure and function of creatine kinase isoenzymes in tissues with high and fluctuating energy demands: the 'phosphocreatine circuit' for cellular energy homeostasis. Biochem. J. 281, 21-40. doi: 10.1042/bj2810021

Wyss, M., and Kaddurah-Daouk, R. (2000). Creatine and creatinine metabolism. Physiol. Rev. 80, 1107-1213. doi: 10.1152/physrev.2000.80.3.1107

Conflict of Interest Statement: The authors declare that the research was conducted in the absence of any commercial or financial relationships that could be construed as a potential conflict of interest.

Copyright (c) 2019 Berry, Schlegel, Kowalski, Bruce, Callahan, Davies-Tuck, Dickinson, Goodson, Slocombe, Snow, Walker and Ellery. This is an open-access article distributed under the terms of the Creative Commons Attribution License (CC BY). The use, distribution or reproduction in other forums is permitted, provided the original author(s) and the copyright owner(s) are credited and that the original publication in this journal is cited, in accordance with accepted academic practice. No use, distribution or reproduction is permitted which does not comply with these terms. 